EUROPEAN ORGANIZATION FOR NUCLEAR RESEARCH

CERN-ISR-TH/71-61

Diffraction Radiation of Charged Rings moving

in a Corrugated Cylindrical Pipe:

A Model of Radiation Losses in an Electron Ring Accelerator

by

E. KEIL

Geneva, 15th December, 1971 


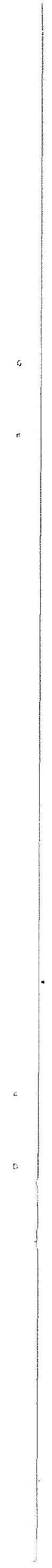


ABSTRACT

The energy loss of a charged ring passing an infinitely long periodic sequence of $R F$ accelerating cavities which has recently received much attention in connection with the collective acceleration of ions in electron rings is calculated by expanding the vector potential into a series of eigenfunctions of the empty waveguide and computing their individual excitation. The frequency spectra obtained are compared to the predictions of simpler analytical models from which a set of formulae for the practical design of $R F$ accelerating structures for electron rings can be derived. The energy loss becomes independent of $\gamma$ when the electron rings have a highly relativistic speed. 


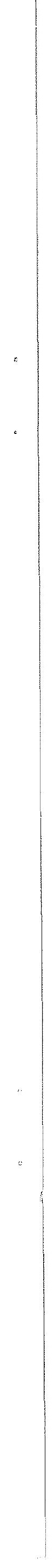


1.

INTRODUCTION

A bunch of charge $Q$ travelling along an $R F$ accelerating structure gains energy due to the accelerating field driven by an external RF source, and it loses energy by exciting additional electromagnetic fields: this is the phenomenon of "beam loading" familiar in linear accelerators ${ }^{1}$ ). The energy gain per unit length $\mathrm{dU} / \mathrm{dz}$ takes the form

$$
\mathrm{dU} / \mathrm{dz}=\mathrm{EQ}-\mathrm{BQ}^{2}
$$

Here $\mathrm{E}$ is the average applied field along the bunch trajectory, and $\mathrm{BQ}^{2}$ is the radiation loss or beam loading term. $\mathrm{B}$ is a proportionality factor whose dependence on the geometrical parameters of the accelerating structure and the bunch is one of the subjects of this paper.

Because of the quadratic increase of the radiation loss with the charge $Q$, the interest in an accurate evaluation of the factor $B$ suddenly rose with the advent of the electron ring accelerator ${ }^{2}$. In fact, the charge of an electron ring is a few orders of magnitude higher than that of a bunch in a typical electron or proton linear accelerator. Consequently, the radiation loss becomes an important factor in the design of an $\mathrm{RF}$ accelerating structure for electron rings. Although the Dubna group ${ }^{2)}$ indicated that the radiation loss does not present an obstacle, some authors 314 ) calculated an increase of the radiation loss with the axial velocity of the electron rings and concluded that there might be a practical upper energy limit for $R F$ acceleration of electron rings.

This discrepancy is due to the different models used in the calculation. Single cavity models 3 (4) show an increase of the radiation loss as $\gamma^{\frac{1}{2}}$. However, their validity breaks down above some value of $\gamma$ when interference between successive cavities becomes an important 
factor, and periodic models are more appropriate. In cylindrical geometry, they yield a radiation loss independent of $\gamma$, and in planar geometry a decrease of the radiation loss as $\left.\gamma^{-\frac{1}{2}} \cdot 5\right)$ A variety of models was employed for the calculation of the radiation loss 6$) 7$ ). We shall represent the accelerating structure (Fig. 1) by an infinitely long periodic sequence of cylindrical cavities of radius $b$ and length $g$, joined by concentric tubes of radius a. The length of a period is $d$. The charge distribution is a uniformly filled ring with rectangular cross-section of length $h$ and radii $r_{1}$ and $r_{2}$. It travels through the pipe at the velocity $v$ from $-\infty$ to $+\infty$.

After a summary of the mathematical approach, numerical results are given for a variety of accelerating structures. They are then compared with the results of other models. From this comparison we derive a set of formulae for the practical design of RF accelerating structures for electron rings.

\section{MATHEMATICAL FORMALISM}

\subsection{Calculation of the energy loss.}

The mathematical formalism employed was developed by Akhiezer, Lyubarskii and Fainberg ${ }^{8}$ ), its application to the present problem is described in 9). We shall give a more transparent derivation which essentially agrees with that used in 10 ).

The field created by the moving charge is obtained from the wave equation for the vector potential $\vec{A}(\vec{r}, t)$ :

$$
\nabla^{2} \vec{A}-\frac{I}{c^{2}} \frac{\partial^{2} \vec{A}}{\partial t^{2}}=-\mu_{0} \vec{J} \quad \ldots(2)
$$


where $\vec{j}$ is the current density related to the moving charge. We shall look for the solution in the form of a sum over the eigen functions of the homogeneous equation (2).

$$
\vec{A}(\vec{r}, t)=\sum_{\lambda} g_{\lambda}(t) \vec{A}_{\lambda}(\vec{r})
$$

The $\vec{A}_{\lambda}$ are normalised as follows :

$$
\int_{V_{N}}\left|\vec{A}_{\lambda}\right|^{2} d V=N / \varepsilon_{0}
$$

where $\mathrm{V}_{\mathrm{N}}=\mathrm{N} \cdot \mathrm{V}$ is the volume of $\mathrm{N}$ cells. Since the boundary is periodic, the eigenfunctions have the form

$$
\vec{A}_{\lambda}(\vec{r})=e^{-i \beta_{0} z} \vec{a}_{\lambda}(\vec{r})
$$

where $\beta_{0}$. is a parameter in the interval $(-\pi / d, \pi / d)$ and $\vec{a}(\vec{r})$ is a periodic function of $\mathrm{z}$ with period $d$. It can therefore be expanded into a Fourier series, and the eigenfunctions become:

$$
\begin{aligned}
\vec{A}_{\lambda}(\vec{r}) & \left.=\sum_{m}^{+\infty} \vec{A}_{\lambda m}(r) \exp -i \beta_{m} z\right) \\
m & =-\infty
\end{aligned}
$$

with the propagation constant $\beta_{m}=\beta_{0}+2 \pi m / d$. The explicit radial dependence can be obtained from (2). We find the z-component, for $r \leqslant a$ :

$$
A_{z \lambda}(r, z)=\frac{i}{\omega}_{m=-\infty}^{+\infty} \sum A_{\lambda m} \frac{J_{0}\left(x_{m} r\right)}{J_{0}\left(x_{m}^{a)}\right.} \exp \left(-i \beta_{m} z\right) \text {. (7) }
$$

with the radial propagation constant $x_{m}^{2}=\omega_{\lambda}^{2} / c^{2}-\beta_{m}^{2}$. The coefficients $A_{\lambda m}$ will be obtained below by field matching.

Because the $\vec{A}_{\lambda}$ are normalised and orthogonal, the field coordinates $q_{\lambda}(t)$ obey the equation:

$$
\ddot{q}_{\dot{\lambda}}+\omega_{\lambda}^{2}{ }_{\lambda}^{q}=\frac{I}{N} \int_{V_{N}} \vec{j} \vec{A}_{\lambda}^{*} d V=f_{\lambda}
$$


We shall limit our calculation to currents flowing in the $\mathrm{z}$ direction, i.e. we consider the case of intense bunches in a linear accelerator for which experimental results ${ }^{l l)}$ are available for comparison. In electron ring accelerators, the azimuthal motion of the electrons in the ring excites $\mathrm{H}$ modes in addition to the $\mathrm{E}$ modes calculated in this paper. It is known, that for an infinite set of half planes ${ }^{5)}$ the energy loss into $\mathrm{H}$ modes is equal to that into $\mathrm{E}$ modes in the limit of $\gamma \rightarrow \infty$.

When the azimuthal motion of the electron is neglected, an electron ring with charge $Q$ and geometrical parameters as shown in Fig. l, travelling with the speed $v$, has the current density:

$$
j_{z}=\frac{Q v}{\pi h\left(r_{2}^{2}-r_{1}^{2}\right)} H\left(\frac{1}{2} h-|z-v t|\right) H\left(r-r_{1}\right) H\left(r_{2}-r\right)
$$

where $H(x)$ is the Heaviside step function, $H(x)=1$ for $x \geqslant 0$ and $H(x)=0$ for $x<0$. Using (7), (9), the right hand side of (8) becomes :

$$
f_{\lambda}(t)=-\frac{Q v i}{N u_{\lambda}} \sum_{m} A_{\lambda m} S\left(\frac{1}{2} \beta_{m} h\right) J\left(x_{m}\right) \exp \left(i \beta_{m} v t\right)
$$

The factors $S(x)=x^{-1} \sin x$ and $J$ take into account the finite dimensions of the electron ring. $J$ is given by :

$$
J\left(x_{m}\right)=\frac{2\left\{r_{2} J_{1}\left(x_{m} r_{2}\right)-r_{1} J_{1}\left(x_{m} r_{1}\right)\right\}}{x_{m}\left(r_{2}^{2}-r_{1}^{2}\right) J_{0}\left(x_{m}^{a}\right)}
$$

With the $N$ cavities centred at $z=0$, and with $j_{z}$ given by (9), $f_{\lambda}(t)$ vanishes for $|t| \geqslant T=\frac{1}{2} \mathrm{Nd} / \mathrm{v}$. Hence, for $t \geq T, q_{\lambda}(t)$ is given by :

$$
q_{\lambda}(t)=\bar{\omega}_{\lambda}^{1} \int_{-T}^{+T} f_{\lambda}\left(t^{\prime}\right) \sin \omega \lambda\left(t-t^{\prime}\right) d t^{\prime}
$$


which becomes:

$$
q_{\lambda}(t)=\frac{Q d}{2 \omega_{\lambda}^{2}} \quad \sum_{m} A_{\lambda m} S\left(\frac{1}{2} \beta_{m} h\right) J\left(\chi_{m}\right)\left\{S\left(\phi^{+}\right) \exp \left(-i \omega_{\lambda} t\right)-S\left(\phi^{-}\right) \exp \left(j \omega_{\lambda} t\right)\right\} \ldots(
$$

where: $\emptyset^{ \pm}=\frac{1}{2} \mathbb{N a}\left(\omega_{\lambda} / v \pm \beta_{m}\right)$ and $S(\varnothing)=\phi^{-1}$ sin $\varnothing$ as above.

For $N \gg 1$, the largest contributions to (13) come from the two terms in the sum with $m=\ell^{ \pm}$for which $\left|\omega_{\lambda} \pm \beta_{\ell \pm}\right|<\pi / N a$.

The summation over the space harmonics $m$ and over the $N$ possible values of $\beta_{0}$, spaced by $2 \pi / N d$, may be combined into a single sum which we write in the form of an integral:

Introducing the new variable $x=\omega_{\lambda} \pm \beta_{m} v$, we find:

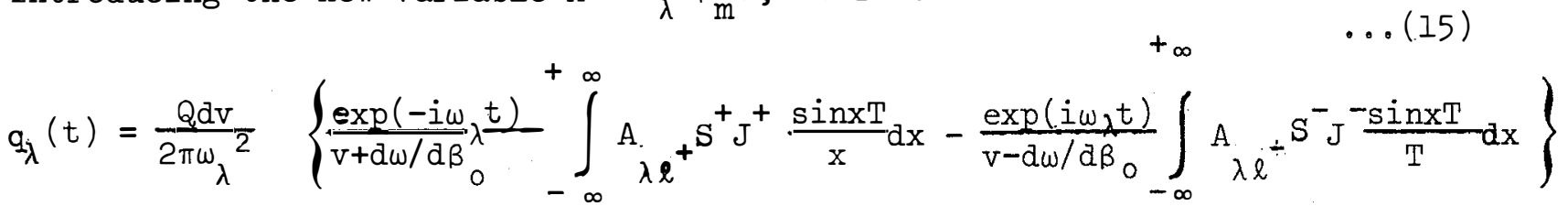

For $\mathrm{T} \rightarrow \infty$, the identity

$$
\lim _{T \rightarrow-\infty}^{+\infty} \int_{-\infty} f(x) \frac{\sin x T}{x} d x=\pi f(0)
$$

may be used. Thus one obtains:

$$
q_{\lambda}(t)=\frac{Q d v}{2 \omega{ }_{\lambda}^{2}}\left\{\frac{\exp \left(-i \omega_{\lambda}\right.}{v+d \omega / d \beta_{0}} A_{\lambda l^{+}} S^{+} J^{+}-\frac{\exp \left(i \omega_{\lambda}\right.}{v-d \omega / d \beta_{0}} A_{\lambda l^{-}} S^{-} J^{-}\right\} \ldots(16)
$$


Here, $A_{\lambda \ell \pm}$ are to be evaluated for $\omega_{\lambda} \pm \beta_{\ell \pm} v=0$. This is achieved by letting $\beta_{0}=u_{\lambda} / v-2 \pi l / d$ with $\ell$ chosen such that $\left|\beta_{0}\right|<\pi / d$. We then find $\beta_{\ell}=\omega_{\lambda} / v$ and $x_{\ell}^{2}=x^{2}=-\omega_{\lambda}^{2} / v^{2} \gamma^{2}$.

Furthermore, it has been verified that ${ }_{\lambda} \lambda \ell^{+=A} \lambda l^{-=f} \lambda l^{\text {which is not }}$ surprising since the change from $\ell^{+}$to $\ell^{-}$just involves changing the sign of $\beta_{0}$ and counting the space harmonics in reversed order. The final answer is :

$\lim q_{\lambda}(t)=-\operatorname{Qdi}_{\lambda}{ }^{-2} A_{\lambda l} S\left(\frac{1}{2} \omega_{\lambda} h / v\right) J\left(i \omega_{\lambda} / \gamma v\right) \sin \omega_{\lambda} t /\left[1-v^{-2}\left(d \omega / d \beta_{0}\right)^{2}\right] \ldots(17)$ $N \rightarrow \infty$

In the following, we shall work to first order in the ratio of the group velocity and the particle velocity. In this case, the term in the square bracket in (17) may be dropped.

The energy loss per period $U_{\lambda}$ is obtained by calculating the stored energy in one cell of the structure and using energy conservation. With the normalisation (4) the stored energy $\mathrm{W}_{\lambda}$ is :

$$
w_{\lambda}=\frac{1}{2}\left(\left|\dot{q}_{\lambda}\right|^{2}+w_{\lambda}{ }^{2}\left|q_{\lambda}\right|^{2}\right)
$$

Substitution from (17) yields, with $U_{\lambda}={ }^{W}{ }_{\lambda}$

$$
U_{\lambda}=\frac{1}{2}\left[{ }^{Q A} \lambda e^{d} S\left(\frac{1}{2} \omega \lambda_{\lambda}^{h / v) J(i \omega / \gamma v) / \omega} \lambda\right]^{2}\right.
$$

This result agrees with that given in 9). 


\section{$2.2 \quad$ Calculation of the field coefficients.}

The suitable field expansion in the tube region $0<r<a$ is given by $(7)$. The propagation constants are $\beta_{0}=\omega / v-2 \pi l / d$ with $\ell$ chosen such that $\left|\beta_{0}\right|<\pi / d, \beta_{m}=\beta_{0}+2 \pi m / d$ and $x_{m}^{2}=\omega^{2} / c^{2}-\beta_{m}^{2}$. In this section we omit the index $\lambda$ because we only consider a single eigenfunction. The tangential field components which are needed for the field matching follow from (16) and from Maxwell's equations.

$$
\begin{gathered}
E_{z}=\sum_{m} A_{m}\left[J_{0}\left(x_{m} r\right) / J_{0}\left(x_{m} a\right)\right] \exp \left(-i \beta_{m} z\right) \\
Z_{0} H_{\varphi}=(i \omega / c) \sum_{m} A_{m}\left[J_{0}\left(x_{m} r\right) / J_{0}\left(x_{m} a\right)\right] \exp \left(-i \beta_{m} z\right)
\end{gathered}
$$

$Z_{0}=120 \pi \mathrm{ohm}$ is the impedance of free space.

In the slot region $a \leqslant r \leqslant b$, we take radial transmission line modes which fulfil all boundary conditions except those at the slot mouth $r=a$ :

$$
\begin{aligned}
& \infty \\
& E_{z}=\sum_{S=0_{\infty}} B_{S} \cos (2 \pi s z / g)\left[F_{\circ}\left(\Gamma_{S} r\right) / F_{0}\left(\Gamma_{S} a\right)\right] \\
& +\sum_{S=I} D_{S} \sin ((2 s-1) \pi z / g)\left[F_{0}\left(\Gamma^{\prime} s_{s} r\right) / F_{0}\left(\Gamma^{\prime} a\right)\right] \\
& \infty \\
& Z_{\circ \varphi} H_{\infty}=(i \omega / c) \sum_{S=0} B_{S} \cos (2 \pi s z / g)\left[F_{I}\left(\Gamma_{S} r\right) / \Gamma_{S} F_{\circ}\left(\Gamma_{S}^{l} a\right)\right] \\
& +(i \omega / c) \sum_{s=1} D_{s} \sin ((2 s-1) z / g)\left[F_{I}\left(\Gamma^{\prime} s^{r}\right) / \Gamma_{s}^{l} F_{0}\left(\Gamma_{s}^{i} a\right)\right]
\end{aligned}
$$


with the propagation constants $\Gamma_{S}^{2}=\omega^{2} / c^{2}-(2 \pi s / g)^{2}$ and $\Gamma_{s}^{\prime 2}=\omega^{2} / c^{2}-((2 s-1) \pi / g)^{2}$ and the functions

$$
F_{i}\left(\Gamma_{r}\right)=Y_{0}(\Gamma b) J_{i}\left(\Gamma_{r}\right)^{i / l}-J_{0}(\Gamma b) Y_{i}(\Gamma r)
$$

The unknown coefficients $A_{m}, B_{S}$ and $D_{S}$ are determined by imposing boundary conditions at $r=a$ : $E_{z}$ must vanish along the tube wall, and $\mathrm{E}_{\mathrm{z}}$ and $\mathrm{H}_{\varphi}$ must be equal acroos the slot mouth ${ }^{12}$ ). Using the fact that the exponential function is orthogonal with period $d$, and that the trigonometric functions are orthogonal with period $g$, we obtain five equations between the unknown coefficients. Eliminating $B_{S}$ and $D_{S}$, we obtain an equation for the $A_{m}$ which can be written in matrix form

$$
\left[\underline{C}^{T} \underline{H} \underline{C} \underline{M}-\underline{S}^{T} \underline{L} \underline{S} \underline{M}-\underline{U}\right] \underline{A}=0
$$

Here $\underline{A}$ is a vector with components $A_{m^{2}} \underline{H}, \underline{L}$ and $\underline{M}$ are diagonal matrices with elements

$$
\begin{aligned}
& \mathrm{H}_{\mathrm{ss}}=\mathrm{K}_{\mathrm{s}} \Gamma_{\mathrm{s}} \mathrm{F}_{0}\left(\Gamma_{\mathrm{s}} \mathrm{a}\right) / \mathrm{F}_{I}\left(\Gamma_{\mathrm{s}} \mathrm{a}\right) \\
& \mathrm{L}_{\mathrm{ss}}=2 \Gamma_{\mathrm{s}} \cdot \mathrm{F}_{0}\left(\Gamma_{\mathrm{s}} \mathrm{a}\right) / \mathrm{F}_{I}\left(\Gamma_{\mathrm{s}} \mathrm{a}\right) \\
& \mathrm{M}_{\mathrm{mm}}=\left(I / d g x_{m}\right) J_{I}\left(x_{m} a\right) / J_{0}\left(x_{m} a\right)
\end{aligned}
$$

where $K_{0}=1$ and $K_{s}=2$ for $s>0 . \underline{C}$ and $\underline{S}$ are matrices with elements

$$
\begin{gathered}
\mathrm{C}_{\text {st }}=(-1)^{\mathrm{s}} \mathrm{g} \frac{\beta_{\mathrm{t}} \mathrm{g}}{2} \sin \frac{\beta_{t^{g}}}{2} /\left[\left(\frac{\beta_{t} \mathrm{~g}}{2}\right)^{2}-\left(\pi_{s}\right)^{2}\right] \\
\left.\mathrm{s}_{\text {st }}=-(-1)^{\mathrm{s}} \text { ig } \frac{\beta_{t} \mathrm{~g}}{2} \cos \frac{\beta_{t} g}{2} /\left[\left(\frac{\beta_{t} g}{2}\right)^{2}+\left(\frac{(2 s-1)}{2}\right)\right)^{2}\right]
\end{gathered}
$$


$\underline{U}$ is the unit matrix and $x^{T}$ is the transpose of $\mathrm{x}$.

The frequencies where (23) has non-trivial solutions are those where wave travelling with the phase velocity $\mathrm{v}$ propagate in the waveguide. Solving (23) gives the coefficients $A_{m}$ apart from a common factor which is determined by the normalisation( 4$)$.

\section{$2.3 \quad$ Numerical procedure}

All the calculations necessary are done by computer. The first program in the chain calculates the determinant of (23) in a given interval of frequency $\omega$, in steps $\Delta \omega$. It finds the frequencies where the determinant vanishes, solves (23) at these frequencies, and normalises the field coefficients $A_{m}$ according to (4). It issues a list of pairs of numbers $\left(\omega, A_{l}\right)$ for use by subsequent programs. The matrices involved are truncated to finite size, which is determined by two considerations. Firstly, there must not be any eigenvalue $\omega$ which gets lost because the relevant space harmonic has been omitted; this means that there must be enough space harmonics so that the propagation constants near the border of the matrix are imaginary. Secondly, there must be enough space harmonics so that $A_{\ell}$ is computed to sufficient accuracy; this is done by trial and error. In the results, the error on the energy loss should not exceed a few percent. In order to avoid confusing the program by the $K$ infinities $\omega_{k}$ of the determinant of (23) in the frequency interval considered, they are suppressed by multiplying the determinant by $\frac{K}{\Pi}\left(1-\omega_{k} / \omega\right)$; the infinities are at the frequencies where $k=1$ 
$F_{I}\left(\Gamma_{S} a\right), F_{I}\left(\Gamma_{S}^{\prime} a\right)$ or $J_{0}\left(x_{m} a\right)$ vanish, which can all be calculated directly. The step-by-step procedure misses zeros which appear in pairs within a step $\Delta \omega$. In order to find them, the expression

$\operatorname{det}(\omega) \prod_{k}^{K}\left(1-\omega / \omega_{k}\right) / \prod_{n=1}^{N}\left(1-\omega / \omega_{n}\right)$ is tabulated, where the $\omega_{n}$ are the $N$ zeros found so far. Missing zeros show up in sharp dips in this function which are found by inspection. These missing zeros are then found, added to the list and the new function is tabulated until it is found to be sufficiently smooth. The size of the truncated matrices is proportional to $w$. This and the fact that the number of zeros in a given frequency interval is also proportional to $\omega^{13)}$ results in a practical upper limit for the frequency which can be handled: beyond it the computational effort involved quickly becomes prohibitive.

The energy loss is calculated from (19) in a program which reads geometrical parameters and pairs $\left(\omega, A_{l}\right)$, sorts them according to geometrical parameters and frequency, and prepares tables and histograms of the frequency distribution of the energy loss, as shown in Figs. $2 \& 3$.

\section{NUMERICAL RESULTS,}

All results are compiled in Table I. All structures are given with dimensions as used in the computation. In principle, the number of geometrical parameters can be reduced by one if the following scaling law is applied: If all geometrical dimensions are scaled by a factor $\alpha$, the resonant frequencies and energy losses are scaled by a factor $1 / \alpha$. The cases 23 to 40 were computed in connection with an electron ring accelerator design study ${ }^{14)}$ at the Lawrence Radiation Laboratory, Berkeley, and the cases 41 to 46 in connection with an experiment on the radiation loss $^{11}$ ) at the Stanford Linear Accelerator Center. 


\section{ANALYTICAL MODELS}

A few models give closed expressions for the radiation loss. They are described below. In Chapter 5, they will be compared with our computational results, and will form the basis of practical formulae for the radiation loss.

\subsection{Lawson's diffraction model}

Lawson has calculated the energy loss using diffraction theory ${ }^{3)}$. He considers diffraction at the up-stream hole of radius a in a cavity of length $g$, and calculates the fraction of energy which is diffracted to radii $r \geqslant$ a at the downstream end wall of the cavity. His result is for a point charge

$$
U=\frac{0.44\left(2 \mathrm{~g} / \pi c^{5}\right)^{\frac{1}{2}} Q^{2} a \gamma^{-2}}{4 \pi \varepsilon 0} \int_{c / a}^{\infty} i^{3 / 2} K_{1}^{2}(\omega a / c \gamma) d \omega
$$

The lower cut-off is introduced in order to allow for the limited validity of the diffraction model. The formula is claimed to hold for $\gamma>1$. The integrand will be used below to draw frequency spectra. Evaluating (26) numerically yields:

$$
U=\frac{0.6}{4 \pi \varepsilon_{0}} \frac{Q^{2} g^{\frac{1}{2}} \gamma^{\frac{1}{2}}}{a^{3 / 2}}
$$

The magnitude of the radiation loss and its increase as $\gamma^{\frac{1}{2}}$ led Lawson to the suggestion that there is a severe practical upper limit to the energy which can be reached by $R F$ acceleration of electron rings.

\subsection{The closed cavity model.}

The resonant frequency $\omega$ is for the $\mathrm{E}_{\text {omn }}$ mode in a simple cylindrical cavity of length $g$ and radius $b$ :

$$
\omega=c\left[\mu_{m}^{2}+(n \pi / g)^{2}\right]^{\frac{1}{2}}
$$


and the energy loss into the $\mathrm{E}$ mode is for an electron rirg of the dimensions shown in Fig. 1 15) 16)17)18) :

$U=\frac{8 Q^{2} \beta^{2}}{4 \pi \varepsilon_{0} g^{2}} \cdot\left(\frac{1+\delta}{2}\right)\left[1-(-)^{n} \cos \frac{\omega g}{v}\right]\left[J\left(\mu_{m}\right) S(\omega h ; 2 v)\right]^{2}\left[1+\left(n \pi / g \mu_{m} \gamma\right)^{2}\right]-2$

$\mu_{m}$ is the $m$ - th solution of $J_{\circ}\left(\mu_{m} b\right)=0$. J is defined as in (Il) with a replaced by $b$ and $S(x)=x^{-1} \sin x$ as before.

Kolpakov and Kotov ${ }^{15)}$ have argued that (29) holds for $\star \geqslant a$ where $a$ is the hole radius. In order to find any modes at all which fulfil this condition, the condition $b / a>j_{1}$ must be satisfied. They have summed (29) over all modes with $\lambda \geqslant a$ in an approximate manner which also requires $g \leqslant 2 \beta a$. Using the same cut-off and the same approximations, Kolpakov, Kotov and Om-San-Kha' have considered the case shown in Fig. 1, and found for a point charge

$$
U=\frac{g^{2}}{4 \pi \varepsilon_{0}} \frac{Q^{2}}{2 a^{2} d}
$$

This is a factor g/d smaller than the result of Kolpakov and Kotov.15) The contributions of all modes were summed up by Winterbon ${ }^{16}$ ) and by Levine and Sessler ${ }^{18)}$. They found that a finite result is only obtained for finite minor ring radius; the result diverges logarithmically for a ring charge with vanishing minor radius, and even more strongly for a point charge. In the case where the axial minor ring dimension is constant in its rest frame, and hence Lorentz contracted in the laboratory frame, the total radiation loss increases without limit with increasing $\gamma$, but less rapidly than $\gamma$.

\subsection{The optical resonator model}

Sessler ${ }^{20)}$ developed a formula for the radiation loss based on the analogy between a set of infinite plates with circular holes and a pair of circular mirrors. The latter case has been treated extensively 
in conjunction with optical resonators ${ }^{21)}$. The argument is as follows : The fractional energy loss $\alpha$ per traversal of the wave between the mirrors, due to the diffraction of the first radial mode, is, according to Vainshtein 21 )

$$
\alpha=8 j_{1}^{2} \sigma(M+\sigma) /\left[(M+\sigma)^{2}+\sigma^{2}\right]^{2}
$$

where $\sigma=\zeta\left(\frac{1}{2}\right) / \sqrt{\pi}=0.824, j_{1}=2.405$ is the first zero of $J_{0}$ and $M=\left(4 \omega_{a}^{2} / c d\right)^{\frac{1}{2}}$.

Sessler found the energy flux Pdw in the frequency interval $\mathrm{d} \omega$ in the beam by evaluating the Poynting vector at the beam edge $r=a$ and multiplying it by the cross-section of the beam :

$$
P d \omega=\frac{Q^{2} a^{2} \omega^{2}}{4 \pi^{2} \varepsilon_{0} \beta^{3} \gamma^{2} c^{3}} K_{1}^{2}(\omega a / \beta \gamma c) d \omega
$$

$\mathrm{K}_{1}$ is the modified Bessel function of the first kind. He obtained the total energy loss from the product of (31) and (32) by integrating over all $\omega$ :

$$
U=\frac{2 j_{1}^{2} \sigma Q^{2} a^{2}}{\pi^{2} \varepsilon \beta^{3} \gamma^{2} c^{3}} \int_{2 \pi c / \alpha}^{\infty} \omega^{2}(M+\sigma) K_{1}^{2}(\omega a / \beta \gamma c)\left[(M+\sigma)^{2}+\sigma^{2}\right]-2 d \omega
$$

He introduced a lower frequency cut-off to allow for the limited validity of diffraction formulae. We have integrated (33) numerically for comparing our computed results with this model. We have also used the integrand for plotting frequency spectra. Closed expressions have been derived by making simplifying assumptions.

For high values of $\gamma, K_{l}(X)$ may be replaced by $I / X$. We then find : 


$$
\left.U=\frac{j_{1}^{2} \sigma d Q^{2}}{2 \pi^{2} \varepsilon_{0} a^{2} \beta} \mid \frac{2 a \sqrt{2 \pi} / d}{(2 a \sqrt{2 \pi} / d+\sigma)^{2}+\sigma^{2}}+\frac{1}{\sigma} \tan ^{-1}\left(\frac{\sigma}{2 a \sqrt{2 \pi} / d+\sigma}\right)\right\} \ldots
$$

This formula can in turn be simplified in two different ways :

i) If $2 a \sqrt{2 \pi} / d \gg \sigma$, i.e. for short periods $d$ and large hole radii a, Sessler found :

$$
U=\frac{j_{1}^{2} \sigma}{4 \pi^{2} \varepsilon_{0}} \quad \sqrt{\frac{2}{\pi}} \frac{a^{2} Q^{2}}{a^{3} \beta}
$$

ii) In the opposite case $2 a \sqrt{2 \pi} / d \ll \sigma$, i.e. for long periods $d$ and small radii a, we find :

$$
\mathrm{U}=\frac{j \dot{I}^{2}}{8 \pi \varepsilon_{0}} \frac{\mathrm{dQ}}{\mathrm{a}^{2} \beta}
$$

All these formulae are for point charges. In this model, the energy loss does not depend on $\gamma$ when $\gamma$ is large; this property and the fact that it describes an infinite periodic structure distinguishes it from the two previous models. To take account of the finite length $h$ of the electron rings or bunches, we introduce an upper frequency cut-off at $\omega=2 \pi \mathrm{c} / \mathrm{h}$. We obtain for high values of $\gamma$ :

$$
\begin{gathered}
U=\frac{j j_{j}^{2} \sigma d^{2}}{2 \pi^{2} \varepsilon_{0}^{a^{2} \beta}}\left\{\frac{\frac{2 a}{d} \sqrt{2 \pi}}{\left(\frac{2 a}{d} \sqrt{2 \pi}+\sigma\right)^{2}+\sigma^{2}}-\frac{2 a \sqrt{\frac{2 \pi}{d h}}}{\left(2 a \sqrt{\frac{2 \pi}{d h}}+\sigma\right)^{2}+\sigma^{2}}\right. \\
\left.+\frac{1}{\sigma} \tan ^{-1} \frac{\sigma\left(2 a \sqrt{\frac{2 \pi}{d}}\right)\left(\frac{1}{\sqrt{h}}-\frac{1}{\sqrt{d}}\right)}{\sigma^{2}+\left(\frac{2 a \sqrt{2 \pi}}{d}+\sigma\right)\left(2 a \sqrt{\frac{2 \pi}{d h}}+\sigma\right)}\right)
\end{gathered}
$$




\section{CONCLUSIONS}

\section{1}

\section{Comparison with an experiment.}

An experiment on the radiation loss has been performed in the electron linear accelerator at $\mathrm{SLAC}^{\mathrm{Il})}$. In this case the bunch length $\mathrm{h}$ was about $1.5 \mathrm{~mm}$. This lead to a reduction of the energy loss, compared to a point charge, by about $40 \%$.

Table II shows a comparison of the computed energy loss and that given in (37). The SLAC structure does not consist of a large number of identical periods, but rather of 10-foot sections inside which the hole radius a and outer radius $b$ vary ${ }^{22}$ ). This fact is ignored in the computation. The first two entries in Table II show an "average" cell, the remaining entries show actual cells at equal intervals along a 10-foot section. The computed energy loss is obtained as follows : The computer results are taken up to $\omega=4$ or $6 \times 10^{11} \mathrm{~s}^{-1}$, and for higher frequencies up to $\omega=c / h$, the same integral as (33) is taken with the appropriate limits, and added to the computer results. The results obtained with the simple formula (37) and by computer are in good agreement, and the experimental result, $38 \mathrm{MeV}$, Is in very good agreement with the calculation.

\subsection{Comparison between computed and analytic results.}

Since the energy loss increases as $\gamma^{\frac{1}{2}}$ in Lawson's model (27), and is constant in the Sessler-Vainshtein model (34), there must be a value of $\gamma$ where the two models yield the same result. Using (27) and (34) we find for the critical $\gamma_{c}$ :

$$
\gamma_{c}=\frac{25 d^{2}}{a g}\left[\frac{2 a \sqrt{2 \pi} / d}{(2 a \sqrt{2 \pi} / d)^{2}+\sigma^{2}}+\frac{1}{\sigma} \tan ^{-1} \frac{\sigma}{2 a \sqrt{2 \pi} / d+\sigma}\right]^{2} \ldots \text { (38) }
$$


Lawson ${ }^{23)}$ suggested that his model applies for $\gamma<\gamma_{c}$, and that the Sessler-Vainshtein model applies for $\gamma>\gamma_{c}$, because at low $\gamma$ and large ratio d/a the electromagnetic fields surrounding the electron ring are matched to the pipe between successive cavities, i.e. there is no interference between cavities. In the opposite case, the interference between successive cavities causes a levelling-off of the energy loss with increasing $\gamma$. Evidence for this was also given by Hemp ${ }^{24)}$, using diffraction theory. The computed results shown in Table I confirm this hypothesis. When $\gamma<\gamma_{c}$ there is an increase of the energy loss with increasing $\gamma$, in the opposite case, $\gamma>\gamma_{c}$, the energy loss is approximately independent of $\gamma$. Further checks on the models are possible by comparing frequency spectra. The most significant checks can be made when $\gamma$ and $\gamma_{c}$ are very different.

Figure 2 shows two examples with $\gamma_{c}>\gamma_{c}$; the frequency spectrum is independent of $\gamma$. Similarly, Figure 3 shows two examples with $\gamma_{c}$; here the frequency spectrum depends on $\gamma$. It is also seen that the two models describe the frequency spectra rather well in their respective range of validity.

The good agreement between computed and analytic frequency spectra even at low frequencies allows us to use the analytic frequency spectra in the whole frequency spectrum. We have adopted the following procedure for calculating the radiation loss from analytic formulae :

i) if $b / a \geqslant j_{1}$, the low frequency contribution is calculated from (30) and the low frequency limit for the remainder of the spectrum is set at $\omega=c / a$. For $b / a<j_{I}$, this limit is taken to be $\omega=c j_{I} / b, i . e$. at the frequency of the lowest closed cavity mode (28).

ii) If $\gamma>\gamma_{c}$ as calculated from (38), the optical resonator model (33) is used with the above lower limit. If $\gamma<\gamma_{c}$, Lawson's model (26) is used with the above limit. 
The energy losses calculated according to this procedure are also shown in Table I. The errors between computed and analytic results are usually below 30\%. The only visible trend in the errors is that Lawson's formula (26) does not seem to hold for very small $\gamma$.

Therefore, the formulae should be sufficiently accurate for a rough parameter survey. Once the range of likely parameters has been narrowed down, more accurate figures for the radiation loss can be obtained by computation for the lower frequency region, and by using analytic formulae beyond that region, choosing the switch-over frequency such that the ratio between the losses in the two ranges becomes reasonable. It may be seen from Table I that in most of our cases the computation could have been stopped at a lower frequency. 
$\mathrm{a}, \mathrm{b}, \mathrm{d}, \mathrm{g}$ are geometrical dimensions shown in Fig. $1 . \gamma_{c}$ is calculated from (38). $U_{c}$ is the computed energy loss up to a circular frequency $\omega, U_{\text {es }}$ is the estimated energy loss for frequencies above $\omega$. $\mathrm{U}=\mathrm{U}_{\mathrm{c}}+\mathrm{U}_{\mathrm{es}} \cdot \mathrm{U}_{\mathrm{th}}$ is the analytic energy loss obtained as described in 5.2. All energy losses are in $\mathrm{keV} /$ electron/cell for point charges of $10^{13}$ particles. The letter code indicates the formulae used for Ues and $U_{t h}$ : KKO means that the low frequency contribution (30) has been included, L stands for Lawson's diffraction model (26), and $S$ for the optical resonator model (33). The last column gives the relative difference between $U$ and $U_{t h}$ in per cent.

$\begin{array}{cccc}\text { a } & \mathrm{b} & \mathrm{d} & {[\mathrm{m}]} \\ .05314 & {[\mathrm{~m}]} & {[\mathrm{m}]} & {[\mathrm{m}]} \\ .05314 & .08314 & .15314 & .07314 \\ .05314 & .08314 & .15314 & .07314 \\ .05314 & .16628 & .15314 & .07314 \\ .05314 & .16628 & .15314 & .07314 \\ .05314 & .16628 & .15314 & .07314 \\ .04000 & .25000 & .02000 & .02060 \\ .06000 & .25000 & .02000 & .02000 \\ .05000 & .20000 & .02000 & .02000 \\ .051500 & .30000 & .02000 & .02000 \\ .05000 & .25000 & .04000 & .02000 \\ .05314 & .16628 & .15314 & .03657 \\ .05314 & .16628 & .07651 & .03657 \\ .05000 & .25000 & .02000 & .02000 \\ .05000 & .25000 & .02000 & .02000 \\ .05000 & .25000 & .020150 & .02000 \\ .02657 & .16628 & .15314 & .07314 \\ .05000 & .25000 & .01000 & .01000 \\ .05000 & .25000 & .03000 & .03000 \\ .05000 & .25000 & .10000 & .10000 \\ .05000 & .25000 & .20000 & .02000 \\ .05000 & .25000 & .10000 & .02000\end{array}$

$\begin{array}{rrr}\gamma & \gamma_{c} & {\left[10^{9}{ }^{-1}\right.} \\ 5 & 108 & 300^{-1} \\ 2 c & 108 & 300 \\ 50 & 108 & 300 \\ 5 & 109 & 100 \\ 20 & 108 & 200 \\ 50 & 108 & 100 \\ 50 & 1 & 200 \\ 50 & 1 & 200 \\ 50 & 1 & 200 \\ 50 & 1 & 260 \\ 50 & 4 & 200 \\ 20 & 216 & 200 \\ 20 & 19 & 200 \\ 20 & 1 & 300 \\ 50 & 1 & 300 \\ 200 & 1 & 300 \\ 20 & 403 & 200 \\ 50 & 1 & 200 \\ 50 & 1 & 200 \\ 50 & 21 & 300 \\ 50 & 1031 & 250 \\ 50 & 107 & 300\end{array}$

$U_{C}$
398
738
856
443
759
695
286
130
181
189
309
557
437
182
191
193
2149
96
274
905
591
517




\begin{tabular}{|c|c|c|c|c|c|c|c|c|c|c|c|c|c|}
\hline NO & & & d & {$[m$} & $\gamma$ & $\gamma_{c}$ & $\left.10{ }^{q_{s}^{\omega}}-1\right]$ & $\mathrm{U}_{\mathrm{C}}$ & U es & $\mathrm{U}$ & $\mathrm{U}_{\mathrm{th}}$ & & ERROR \\
\hline 23 & .19000 & .50000 & .15500 & .03200 & 40 & 9 & 100 & 66 & 0 & 66 & 73 & $S+K K O$ & 9 \\
\hline 24 & .19000 & .50000 & .07600 & .01900 & $4 C$ & 1 & 100 & 36 & 0 & 36 & 30 & $S+K K C$ & 17 \\
\hline 23 & .19000 & .50000 & .21000 & .03200 & $4 C$ & 29 & 100 & 75 & 1 & 75 & 103 & $S+K K O$ & 27 \\
\hline 26 & .24000 & .50000 & .15500 & .03200 & 40 & 5 & 100 & 41 & 0 & 41 & 40 & $S$ & 3 \\
\hline 27 & .24000 & .50000 & .01600 & .01900 & 40 & 1 & 100 & 21 & 0 & 21 & 16 & $S$ & 26 \\
\hline 78 & .24000 & .50000 &.$? 1000$ & . (1) 3200 & 40 & 15 & 100 & 50 & 0 & 50 & 58 & $S$ & 13 \\
\hline 29 & .14000 & .50000 & $.155 n \mathrm{i}$ & .03200 & 40 & 21 & $10 n$ & 112 & 2 & 114 & 142 & $S+K K C$ & 19 \\
\hline 30 & .140000 & .50000 & .07600 & .01900 & 40 & 2 & 100 & 63 & 1 & 64 & 60 & $S+K K O$ & 6 \\
\hline 31 & .14000 & - 51.)(1000 & .21000 & .03200 & 40 & 66 & 100 & 130 & 15 & 146 & 164 & $L+K K C$ & 11 \\
\hline 32 & .19000 & .50000 & .15500 & .03200 & 20 & 9 & 100 & 58 & 0 & 58 & 62 & $S+K K O$ & 7 \\
\hline 33 & .19000 & .50000 & .07600 & .01900 & $2 C$ & 1 & 100 & 33 & 0 & 33 & 26 & $S+K K C$ & 21 \\
\hline 34 & .19000 & .50000 & .21000 & .03200 & 20 & 27 & 100 & 64 & 0. & 64 & 67 & $L+K K C$ & 5 \\
\hline 35 & .24000 & .50000 & .15500 & .03200 & 20 & 5 & 100 & 35 & 0 & 35 & 34 & $S$ & 4 \\
\hline 36 & .24000 & .50000 & .07600 & .01900 & 20 & 1 & 100 & 19 & 0 & 19 & 14 & $S$ & 29 \\
\hline 37 & .24000 & .50000 & .21000 & $.032 \mathrm{Co}$ & 20 & 15 & 100 & 43 & 0 & 43 & 49 & $S$ & 1.2 \\
\hline 38 & $.140,00$ & .50000 & .13500 & .03200 & 20 & 21 & 100 & 100 & 1 & 101 & $1 \cup 7$ & $L+K K C$ & 5 \\
\hline 39 & .14000 & .50000 & .07600 & .01900 & 20 & 2 & 100 & 59 & 0 & 59 & 52 & $S+K K C$ & 11 \\
\hline 41$)$ & .14000 & .50000 & .21000 & .03200 & 20 & 66 & 100 & 114 & 1 & 115 & $1 C 6$ & $L+K K O$ & $\varepsilon$ \\
\hline 41 & .01152 & .04167 & .03500 & .02911 & 999 & 70 & 600 & 4617 & 2563 & 7183 & 8950 & $S+K K O$ & 20 \\
\hline 42 & .01152 & .04167 & .03500 & .02911 & 4000 & 70 & 400 & 4162 & 3524 & 7686 & 9343 & $S+K K C$ & 18 \\
\hline 43 & .01294 & .04198 & .03500 & .02911 & 4000 & 54 & $4 \mathrm{CO}$ & 3453 & 2546 & 6001 & $72 \times 8$ & $S+K K C$ & 18 \\
\hline 44 & .01226 & .04179 & .03500 & .02911 & 4000 & 61 & 400 & 3762 & 2962 & 6724 & 8180 & $S+K \times C$ & 18 \\
\hline 45 & .01142 & .04158 & .03500 & .02911 & 4000 & 71 & 400 & 4212 & 30,10 & 7823 & 9518 & $S+K K O$ & 18 \\
\hline 46 & .01039 & .04135 & .03500 & .02911 & 4000 & 88 & 400 & 4833 & 4689 & 9524 & 11638 & $S+K K C !$ & 18 \\
\hline
\end{tabular}




\section{Table II}

Comparison of calculated energy losses in $\mathrm{MeV}$ for the SLAC experiment: $10^{9}$ electrons, $\mathrm{r}=\mathrm{h}=1.5 \mathrm{~mm}, 86000$ cells, $\mathrm{d}=3.5 \mathrm{~cm}, \mathrm{~g}=2.9112 \mathrm{~cm}$.

$\begin{array}{clccc}\mathrm{a}(\mathrm{cm}) & \mathrm{b}(\mathrm{cm}) & \gamma & \begin{array}{l}\text { computed } \\ \text { energy loss }\end{array} & \begin{array}{l}\text { energy loss } \\ \text { from (37) }\end{array} \\ 1.152 & 4.1665 & 999 & 37.9 & 41.3 \\ 1.152 & 4.1665 & 4000 & 38.5 & 41.3 \\ 1.294 & 4.198 & 4000 & 31.7 & 31.7 \\ 1.226 & 4.179 & 4000 & 34.7 & 35.9 \\ 1.142 & 4.158 & 4000 & 39.0 & 42.2 \\ 1.039 & 4.135 & 4000 & 45.2 & 52.0\end{array}$




\section{References}

1) P.M. Lapostolle and A.L. Septier, ed., Linear Accelerators, North Holland, Amsterdam (1970).

2) V.I. Veksler et al., Proc. Int. Conf. High Energy Acc., Cambridge, Mass., 289 (1967).

3) J.D. Lawson, Rutherford High Energy Lab. Report/M144 (1962)(unpublished).

4) G.V. Voskresenskii and V.N. Kurdjumov, Proc. Int.Conf. High Energy Acc., Yerewan, 1969, Vol. II, 568.

5) R.D. Hazeltine, M.N. Rosenbluth and A.M. Sessler, J. Math. Phys. 12, 502 (1971)

6) J.D. Lawson, Proc. Int. Conf. High Energy Acc., Yerewan 1969, Vol. II, 463.

7) B. Schnizer, Proc. 4th Work Meeting on Electron Ring Accelerators, Gardening Report IPP-0/3, 158 (1971) (unpublished).

8) A.I. Akhiezer, G. Ya. Lyubarskij, Ya.B.Fainberg, J. Techn. Phys. USSR 25, 2526 (1955).

9) E. Keil, Proc. Int. Conf. High Energy Acc., Yerewan 1969, Vol. II, 551.

E. Keil, C. Pellegrini and A.M. Sessler, Nucl. Instrum. Methods 25, 131 (1971).

11) R.F. Koontz, G.A. Loew and R.H. Miller, Proc. Int. Conf. High Energy Acc., Geneva, 491 (1971)

12) W. Walkinshaw and J. S. Bell, A.E.R.E. G/R 675 (1951) (unpublished)

13) R. Courant and D. Hilbert, Methods of Mathematical Physics, Vol. I, 432, Interscience, New York (1953).

14) Conceptual Studies for New Technology Proton Accelerators, Lawrence Radiation Laboratory (1970) (unpublished) 
15) O.A. Kolpakov and V.I. Kotov, Sov. Phys. Techn. Phys. 2, 1072 (1965)

16) K.B. Winterbon, Atomic Energy of Canada Ltd. Report FSD/ING-16, Chalk River, Ontario (1965) (unpublished)

17) P.L. Morton and V.K. Neil in Proc. of the Electron Ring Accelerator Symposium, Lawrence Radiation Laboratory Report UCRL-18103, 365 (1968) (unpublished).

18) B.J. Levine and A.M. Sessler, Proc. 1969 Particle Accelerator Conference, IEEE Transactions on Nuclear Science NS-16, Vol. 3, 1031 (1969).

19) O.A. Kolpakov, V.I. Kotov and Om-San-Kha, Sov. Phys.Techn. Phys. 10, 18 (1965).

20) A.M. Sessler, private communication (1969).

21) L.A. Vainshtein, Sov. Phys. JETP 17, 709 (1963).

22) R.B. Ne I, ed., the Standard Two-Mile Accelerator,

W.A. Benjamin, New York (1968).

23) J.D. Lawson, private communication (1970).

24) J. Hemp, thesis, Oxford University, Engineering Dept. (1970). 


\section{FIGURE CAPTIONS}

Fig. 1 Three periods of the waveguide with geometrical parameters. The geometrical parameters of the electron ring are also indicated.

Fig. 2 Energy loss spectrum for $\gamma>\gamma_{c}$ as given in (38). The left graph shows case no. 15, the right graph shows case no. 16 in Table $I . L$ is the energy loss spectrum (26) according to Lawson, S-V that according to Sessler-Vainshtein (33).

Fig. 3 Energy loss spectrum for $\gamma<\gamma_{c}$ as given in (38). The left graph shows case no. 40, the right graph shows case no. 31 in Table I. $L$ is the energy loss spectrum (26) according to Lawson, $S-V$ that according to Sessler-Vainshtein (33). 


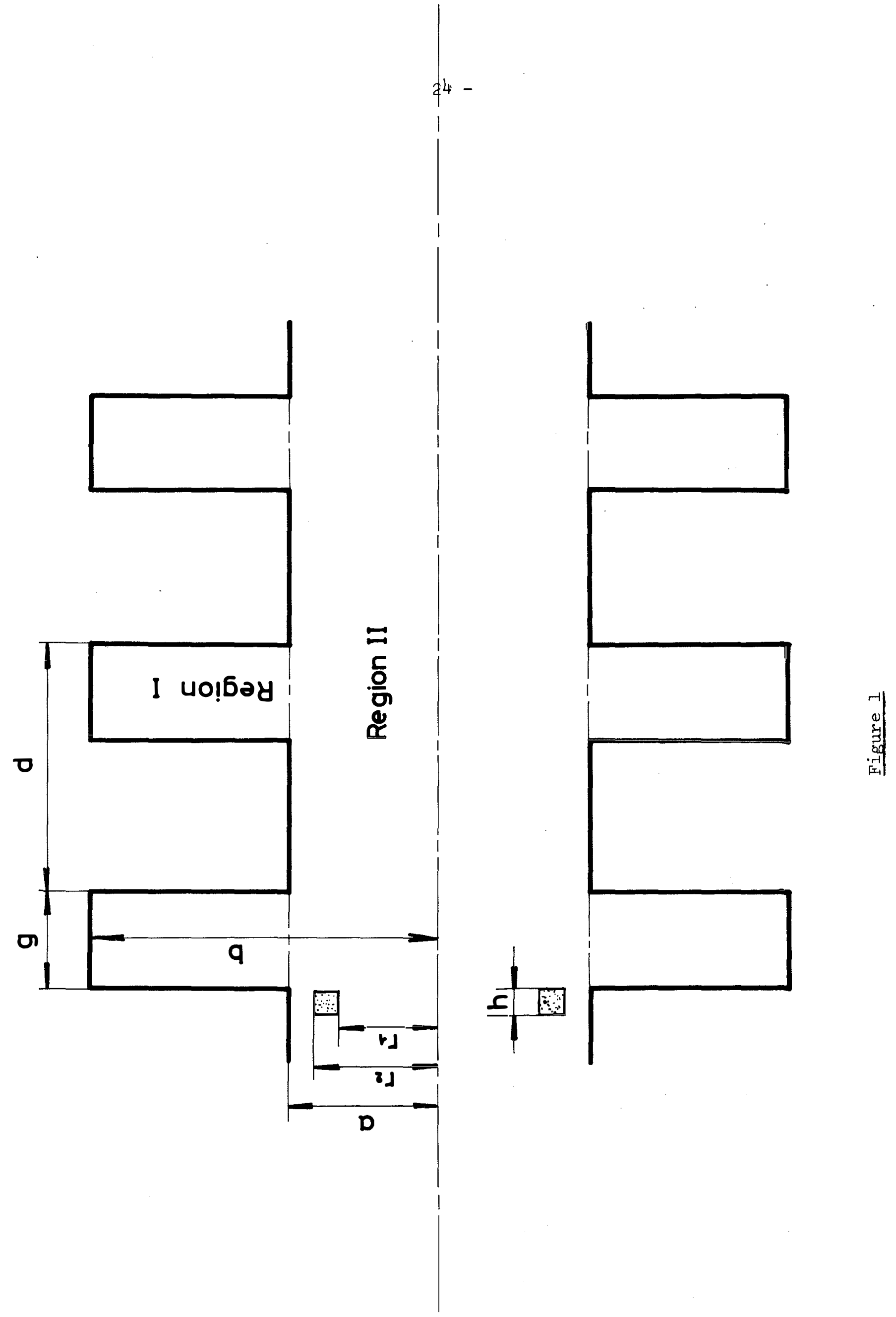




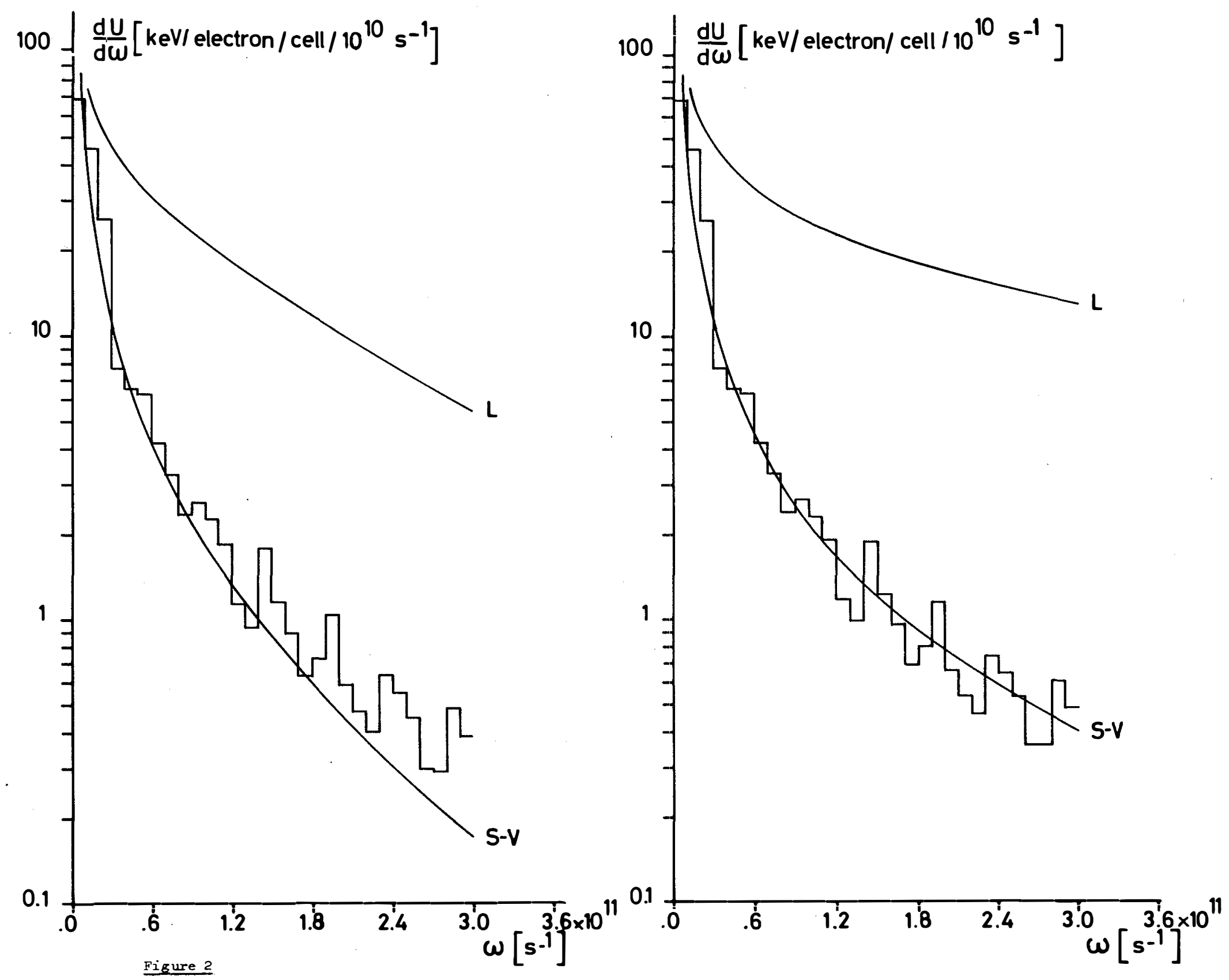



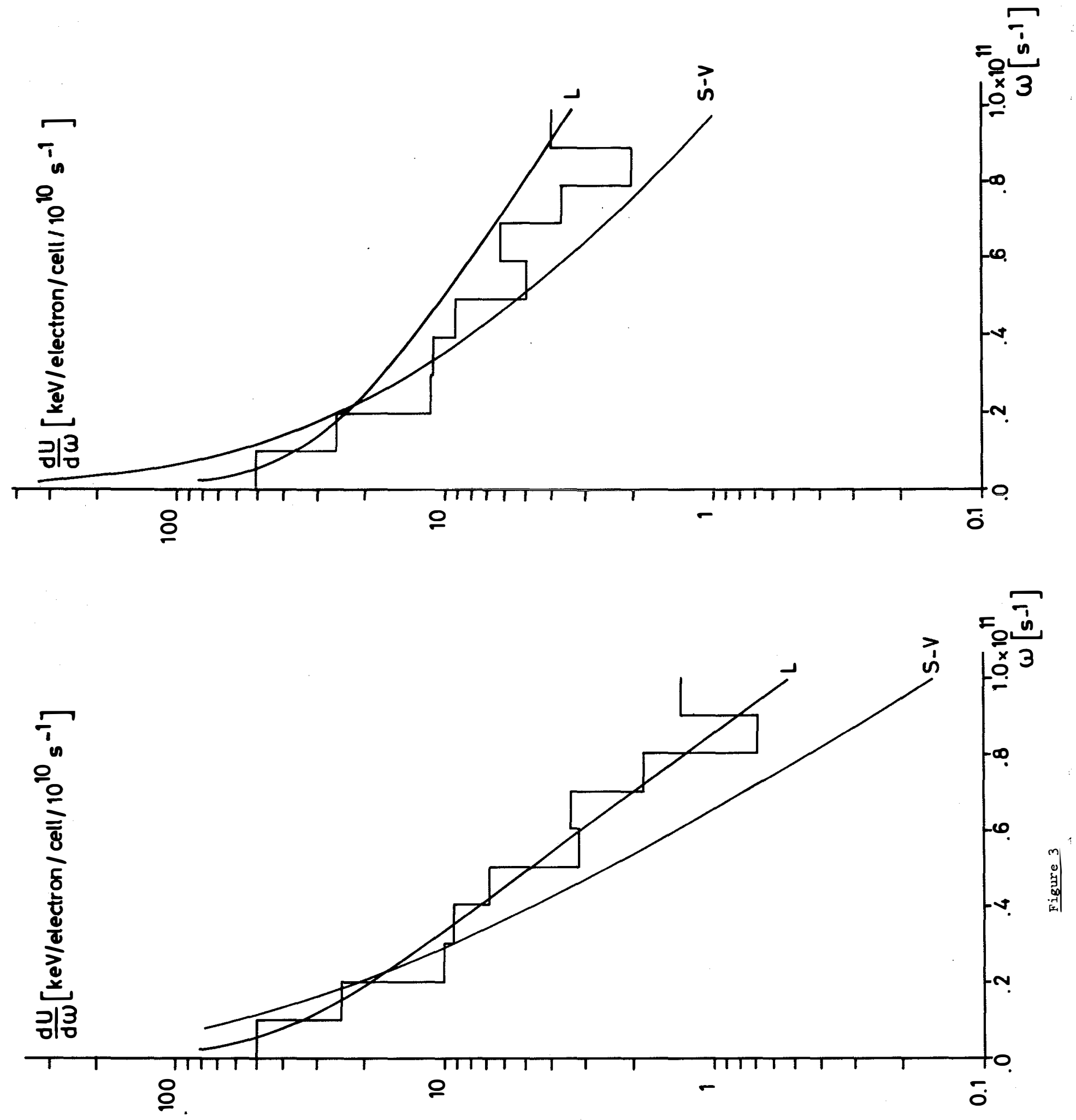
